\title{
Long-term strategies for thyroid health monitoring after nuclear accidents: recommendations from an Expert Group convened by IARC
}

An international multidisciplinary Expert Group convened by the International Agency for Research on Cancer (IARC) evaluated scientific evidence to formulate recommendations about long-term strategies for thyroid health monitoring after a nuclear power plant accident. The work of the Expert Group was published as IARC Technical Publication No. 46. ${ }^{1}$ The objective of this Expert Group was not evaluation of the thyroid examination programmes that were implemented after the past nuclear accidents, or recommendations related to thyroid health monitoring activities currently in progress.

Since the Chernobyl accident in 1986 in Ukraine, the guidelines for overall preparedness and response to nuclear emergencies have evolved ${ }^{2}$ and have contributed to the implementation of successful countermeasures against radiation exposure from nuclear accidents and associated potential adverse health effects. ${ }^{3}$ In view of the established association of thyroid cancer risk with radiation exposure, particularly during childhood and adolescence, appropriate preparedness and response regarding thyroid cancer-related issues are crucial. However, currently, no specific guidelines are available on thyroid cancer screening in populations affected by nuclear accidents.

Previous nuclear power plant accidents, at Three Mile Island (PA, USA) in 1979, Chernobyl, and Fukushima Daiichi (Japan) in 2011, were quite different to each other in terms of the quantity of radionuclides released, exposure pathways, and average levels of radiation exposure to the thyroid, as well as interventions administered. After the Fukushima Daiichi and Chernobyl accidents, affected children and adolescents underwent thyroid examinations, but the implemented programmes differed in the size of the population and the length of time since the accident. Although questions remain about the potential public health benefits of thyroid screening programmes, the extensive efforts which were made after the respective accidents yielded knowledge and lessons learned that are invaluable in guiding preparations for any future nuclear accidents.

The guiding principle for any health intervention is to maximise benefit and minimise harm, and this approach should also be applied to thyroid health monitoring after nuclear power plant accidents. With this concept in mind, the Expert Group developed the following two recommendations on thyroid health monitoring after possible future nuclear accidents. First, the Expert Group recommends against population thyroid screening after a nuclear accident, and second, it recommends that consideration be given to offering a long-term thyroid monitoring programme for higher-risk individuals (ie, those exposed in utero or

during childhood or adolescence to a thyroid dose of Lancet Oncol 2018 $\geq 100-500 \mathrm{mGy}$ ) after a nuclear accident.

For the first recommendation, population thyroid screening is herein defined as actively recruiting all residents of a defined area, irrespective of exposure level, to participate in thyroid examinations followed by clinical management according to an established protocol. The Expert Group recommends against population thyroid screening after a nuclear accident, because the harms outweigh the benefits at the population level. Although cancer screening can be a valuable public health strategy for improving population health, the extents of benefits and harms vary depending on several factors, including types of cancer, target populations, screening modalities and frequency, resources, and social values. Because thyroid cancer has a large reservoir of subclinical disease in the population, ${ }^{4}$ population thyroid screening identifies cancers that would have developed into clinical cases as well as those that would not have been diagnosed if the screening had not taken place or would not have caused symptoms or death during the patient's lifetime. For example, in South Korea, ${ }^{5}$ the incidence of thyroid cancer increased dramatically without substantial change in the already low disease-specific mortality as use of thyroid ultrasonography examination in clinical practice increased. This phenomenon observed in the South Korea and other countries raised concerns about overdiagnosis, ${ }^{6}$ which often leads to medical interventions with potential risks of complications and negative psychosocial effects.

In the context of overdiagnosis and low diseasespecific mortality, recommendations have been devel-
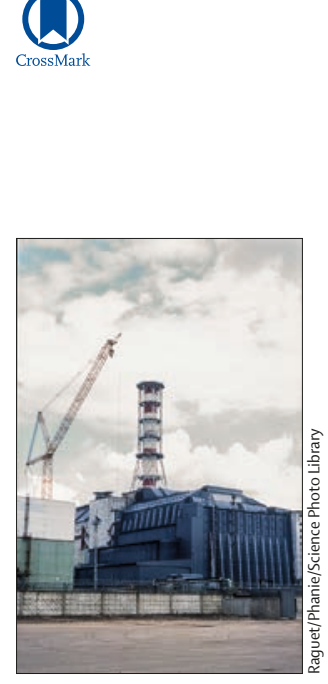
oped by professional organisations to avoid thyroid ultrasonography screening in low-risk, asymptomatic adult populations. ${ }^{8}$ The Expert Group agrees with this view for populations, of all ages, affected by nuclear accidents, because screening the affected asymptomatic populations irrespective of risk levels (ie, thyroid radiation dose) is also expected to result in the issues related to overdiagnosis without clear public health benefits.

For the second recommendation regarding a longterm monitoring programme, thyroid monitoring programme is herein defined as including education to improve health literacy, registration of participants, and centralised data collection from thyroid examinations and clinical management. Thyroid monitoring is an elective activity offered to higher-risk individuals, who might choose how and whether to undergo thyroid examinations in an effort to benefit from early detection and treatment of less advanced disease. Although the aim in both screening and monitoring programmes is early detection of cancer in asymptomatic individuals, the public health approach and objectives are different. In a thyroid monitoring programme, the starting point is the individual instead of the population; higher-risk individuals are offered the programme rather than actively recruited to the programme, and they are empowered to make an informed decision consistent with their values, preferences, and context.

Although some data have shown clinical usefulness of screening for differentiated thyroid cancer in highrisk populations (eg, for familial non-medullary thyroid cancer) ${ }^{9}$ the evidence for benefits of early diagnosis is currently scarce in children. ${ }^{10}$ In view of the risks related to overdiagnosis, the potential consequences of thyroid examinations should be discussed between the affected families and clinicians within the WHO Framework on integrated people-centred health services. Well informed individuals who perceive that potential benefits outweigh potential harms for themselves should receive highquality services from qualified medical professionals in an organised monitoring programme, with governmental authority oversight and quality assurance within an achievable and sustainable financing strategy.

Higher-risk individuals who might benefit from a thyroid monitoring programme after a nuclear accident are defined herein by the Expert Group as those exposed in utero or during childhood or adolescence with a thyroid dose of 100-500 mGy or more. This range of
100-500 mGy was proposed by the Expert Group as a practical definition of an actionable level to offer inclusion into the thyroid monitoring programme. This actionable level should not be confused with radiation protection limits. The choice of a thyroid dose range reflects the option to be more inclusive (lower actionable levels) or to be more efficient (higher actionable levels) in monitoring and identifying radiation-associated thyroid disease in individuals at elevated risk. The Expert Group acknowledges that further research is necessary and the optimal actionable level might need to be revised as new evidence emerges.

Notably, the recommendation of establishing a thyroid dose actionable level does not mean that nothing should be offered to an individual below this exposure level. Despite the potential harms, some low-risk individuals with fears about thyroid cancer might opt to undergo thyroid examinations to seek reassurance. Low-risk individuals who are willing to have a thyroid examination after receiving a detailed explanation of potential benefits and harms should be offered a thyroid examination within the framework of the organised thyroid monitoring programme.

Any thyroid monitoring programme for higher-risk individuals should be initiated as soon as it is practically feasible and should extend through adulthood, given the evidence that thyroid cancer risk from radiation exposure during childhood or adolescence continues into adult life. Intervals between individual thyroid examinations might range between 2 years and 5 years and can be adjusted on the basis of clinical findings and screening modalities. Benefits and harms of thyroid examinations should be balanced against the presence of comorbidities, and a decision to stop should be an informed individual choice.

The recommendations were developed in the context of considerations relevant to exposure to any toxic (including radioactive) substances, and preparedness and response to nuclear accidents, given their implications for decision making about thyroid health monitoring. The considerations include the establishment of a health surveillance programme, including cancer registration, as well as a dynamic risk communication programme, in the vicinity of nuclear installations before a nuclear accident, and also implementing timely and appropriate active dosimetry monitoring and protective actions, such as an iodine thyroid blocking programme, immediately after a 
nuclear accident.

The Expert Group acknowledges that there might be important considerations in addition to the scientific evidence during such decision making processes, including socioeconomic implications, health-care resources, and social values, and that the final decisions are made by the government, the relevant authorities, and the society affected by the nuclear accident. These recommendations are intended to serve as a reference primarily for government officials, policy makers, and health professionals who would be involved in the decision making, planning, and implementation of thyroid monitoring in case of a nuclear accident.

\section{Kayo Togawa, Hyeong Sik Ahn, Anssi Auvinen,} Andrew J Baver, Juan P Brito, Louise Davies, Ausrele Kesminiene, Dominique Laurier, Evgenia Ostroumova, Furio Pacini, Christoph Reiners, Sergey Shinkarev, Geraldine Thomas, Mykola Tronko, Salvatore Vaccarella, *Joachim Schüz

Section of Environment and Radiation (KT, AK, EO, JS) and Section of Infections (SV), International Agency for Research on Cancer (IARC), Lyon, France; Department of Preventive Medicine, College of Medicine, Korea University, Seoul, South Korea (HSA); Epidemiology, Faculty of Social Sciences, University of Tampere, Tampere, Finland (AA); Division of Endocrinology and Diabetes, Department of Pediatrics, Children's Hospital of Philadelphia, Perelman School of Medicine, The University of Pennsylvania, Philadelphia, PA, USA (AJB); Division of Endocrinology, Department of Medicine, Mayo Clinic, Rochester, MN, USA (JPB); Department of Surgery-Otolaryngology-Head and Neck Surgery, The Dartmouth Institute for Health Policy and Clinical Practice, Geisel School of Medicine, Hanover, NH, USA (LD); VA Outcomes Group, Department of Veterans Affairs Medical Center, White River Junction, VT, USA (LD); Division of Health and Environment, Institute for Radiological Protection and Nuclear Safety, Fontenay-aux-Roses, France (DL); Department of Medical, Surgical and Neurological Sciences, Università di Siena, Siena, Italy (FP); Department of Nuclear Medicine, University Hospital Würzburg, Würzburg, Germany (CR); Department of Industrial Radiation Hygiene, State Research Center-Burnasyan Federal Medical Biophysical Center, Moscow, Russia (SS); Department of Surgery and Cancer, Imperial College London, London, UK (GT);
Department of Fundamental and Applied Problems of Endocrinology, Institute of Endocrinology and Metabolism of Ukraine's National Academy of Sciences, Kyiv, Ukraine (MT) schuzj@iarc.fr

DL reports that his institution, Institute for Radiological Protection and Nuclear Safety, benefits from research funding from Areva and EDF. GT reports having received support for travel from TEPCO. All other authors declare no competing interests. The technical report summarised in this Comment (which was released on Sept 29,2018 ) was jointly written by all authors, and discussed at two meetings on Oct 23-25, 2017, and Feb 21-23, 2018, in Lyon, France. The Comment was drafted by KT and JS, and critically reviewed and finalised by all authors. We thank Zhanat Carr, André Ilbawi, and Hiroki Shimura (specialists), and Enora Clero, Silvia Franceschi, Maria Perez, and Catherine Sauvaget (advisers) for their critical input during the discussions and synthesis of the scientific evidence base for the recommendations. We also thank Catherine Chassin (IARC Section of Environment and Radiation) for her skilful administrative support, Jennifer Brandt for editing the report, and the production team of Karen Müller and Sylvia Lesage (IARC Communications Group) for checking and formatting the report. Last, we express our gratitude to the Ministry of the Environment, Japan, for their financial support of the project and to the Nuclear Safety Research Association of Japan for managing the grant. The funder had no involvement in developing the recommendations or in the writing of this article. JPB receives funding from the Karl-Erivan Haub Family Career Development Award in Cancer Research at Mayo Clinic in Rochester (MN, USA), honouring Richard F Emslander. The work of LD was supported by the US State Department (Fulbright grant) and the US Department of Veterans Affairs.

1 IARC Expert Group on Thyroid Health Monitoring after Nuclear Accidents (2018). Thyroid health monitoring after nuclear accidents. IARC Technical Publication No. 46. Lyon: International Agency for Research on Cancer (in press)

2 IAEA. Preparedness and response for a nuclear or radiological emergency: general safety requirements (No. GSR Part 7). 2015. https://www-pub.iaea. org/MTCD/Publications/PDF/P_1708_web.pdf (accessed Sept 6, 2018).

3 IAEA. The Fukushima Daiichi accident: radiological consequences. 2015. https://www-pub.iaea.org/MTCD/Publications/PDF/AdditionalVolumes/ P1710/Pub1710-TV4-Web.pdf (accessed Sept 6, 2018).

4 Furuya-Kanamori L, Bell KJ, Clark J, Glasziou P, Doi SA. Prevalence of differentiated thyroid cancer in autopsy studies over six decades: a meta-analysis. J Clin Oncol 2016; 34: 3672-79.

5 Ahn HS, Kim HJ, Kim KH, et al. Thyroid cancer screening in South Korea increases detection of papillary cancers with no impact on other subtypes or thyroid cancer mortality. Thyroid 2016; 26: 1535-40.

6 Vaccarella S, Franceschi S, Bray F, Wild CP, Plummer M, Dal Maso L. Worldwide thyroid-cancer epidemic? The increasing impact of overdiagnosis. N EnglJ Med 2016; 375: 614-17.

7 Perrier ND, Brierley JD, Tuttle RM. Differentiated and anaplastic thyroid carcinoma: major changes in the American Joint Committee on Cancer eighth edition cancer staging manual. CA Cancer J Clin 2018; 68: 55-63.

8 Bibbins-Domingo K, Grossman DC, Curry S), et al. Screening for thyroid cancer: US Preventive Services Task Force recommendation statement. JAMA 2017; 317: 1882-87.

9 Klubo-Gwiezdzinska J, Yang L, Merkel R, et al. Results of screening in familial non-medullary thyroid cancer. Thyroid 2017; 27: 1017-24.

10 Clement SC, Kremer LCM, Verburg FA, et al. Balancing the benefits and harms of thyroid cancer surveillance in survivors of childhood, adolescent and young adult cancer: recommendations from the international Late Effects of Childhood Cancer Guideline Harmonization Group in collaboration with the PanCareSurFup Consortium. Cancer Treat Rev 2018; 63: 28-39. 